\title{
Linear Orders in the Pushdown Hierarchy
}

\author{
Laurent Braud and Arnaud Carayol \\ LIGM, Université Paris-Est \& CNRS \\ $\{$ braud, carayol\}@univ-mlv.fr
}

\begin{abstract}
We investigate the linear orders belonging to the pushdown hierarchy. Our results are based on the characterization of the pushdown hierarchy by graph transformations due to Caucal and do not make any use of higher-order pushdown automata machinery.

Our main results show that ordinals belonging to the $n$-th level are exactly those strictly smaller than the tower of $\omega$ of height $n+1$. More generally the Hausdorff rank of scattered linear orders on the $n$-th level is strictly smaller than the tower of $\omega$ of height $n$. As a corollary the Cantor-Bendixson rank of the tree solutions of safe recursion schemes of order $n$ is smaller than the tower of $\omega$ of height $n$.

As a spin-off result, we show that the $\omega$-words belonging to the second level of the pushdown hierarchy are exactly the morphic words.
\end{abstract}

\section{Introduction}

The pushdown hierarchy (also known as the Caucal hierarchy) is a hierarchy of families of infinite graphs having decidable monadic second-order (MSO) theory. This hierarchy has several equivalent definitions and has received a lot of attention in the last ten years (see Tho03, Ong07 for surveys).

At the first level of this hierarchy are the transition graphs of the pushdown automata which coincide with the prefix-recognizable graphs [Cau96. Every level can in a similar fashion be characterized as the transitions graphs of an extension of pushdown automata called an higher-order pushdown automata Mas76. The rewriting approach of Cau96 was also extended to all levels in Car05. The deterministic trees at level $n$ correspond to the (trees solutions of) safe recursion schemes of order $(n-1)$ Cau02, KNU02.

An alternative characterization due to Caucal [Cau02, CW03] consists in constructing these graphs by applying MSO-interpretations and graph unfoldings starting from finite trees. The level of a graph in this approach is given by the number of times the unfolding operation has been applied.

Despite these various characterizations, one of the main question concerning this hierarchy is to characterize the graphs inhabiting each level and in particular to provide tools for showing that a structure does not belong to a certain level. For instance, to the authors knowledge, the only proof of the strictness of the pushdown hierarchy relies on the strictness of the hierarchy of languages accepted by higher-order pushdown automata obtained in Eng91. A partial answer is given in Blu08 which provides a pumping lemma for these automata. However

S. Abramsky et al. (Eds.): ICALP 2010, Part II, LNCS 6199, pp. 88 992010.

(C) Springer-Verlag Berlin Heidelberg 2010 
the bounds provided in the latter article are not tight and hence do not allow to derive the strictness level by level.

This article subscribes to this line of research and characterizes linear orders in the pushdown hierarchy.

Linear orders in this hierarchy have first been studied via recursion schemes. A natural way to define a linear order starting from an ordered tree is to consider its frontier i.e. its set of leaves ordered lexicographically. This approach was initiated by Courcelle in Cou78. Two families of linear orders are of particular interest : the well-orders (or ordinals) and the scattered orders.

At the first level of the hierarchy, the scattered frontiers of the regular deterministic tree (order-0 schemes) have an Hausdorff rank strictly less than $\omega$ [Hei80] and the ordinals are known to be those strictly smaller than $\omega^{\omega}$.

At the second level, the frontiers of order- 1 recursion schemes are also called algebraic linear orders. Is was shown in BÉ07, BÉ10 that algebraic ordinals are precisely the ordinals strictly smaller than $\omega^{\omega^{\omega}}$. In BÉ09, it is shown that any scattered algebraic linear order has a Hausdorff rank strictly smaller than $\omega^{\omega}$. They conjecture that similar bounds can be obtained for recursion schemes of arbitrary orders. The results presented in this article prove this conjecture in the case of safe recursion schemes. Our main tool is the characterization of the pushdown hierarchy in terms of graph transformations.

In Section 3, we show that any linear order at level $n$ is isomorphic to the frontier of some order- $(n-1)$ safe recursion scheme. We use this result to show that the $\omega$-words on the second level are exactly the morphic words. In Section 4 . we show that ordinals at level $n$ are exactly the ordinals below $\omega \uparrow \uparrow(n+1)$ (which stands for the tower of $\omega$ of height $n+1$ ). The fact that all these ordinals are in at level $n$ was shown in Bra09. This result provides a self-contained proof of the strictness of the pushdown hierarchy level by level. In Section [5, we show that the Hausdorff rank of scattered orders on level $n$ is strictly smaller than $\omega \uparrow \uparrow n$. As a corollary we obtain that the Cantor-Bendixson rank of the tree solutions of safe recursion schemes of order $n$ is smaller than $\omega \uparrow \uparrow n$.

\section{Preliminaries}

\subsection{Linear Orders and Trees}

A linear order $L=(D,<)$ is given by a set $D$ together with a total order $<$ on $D$. We write $L^{*}$ for the linear order $\left(D,<^{*}\right)$ where $x<^{*} y$ iff $y<x$. For a detailed presentation of linear orderings, we refer to Ros82. $L_{1}=\left(D_{1},<_{1}\right)$ is a subordering of $L_{2}=\left(D_{2},<_{2}\right)$, written $L_{1} \preceq L_{2}$, if $D_{1} \subseteq D_{2}$ and $<_{1}$ is equal to $<_{2}$ restricted to $D_{1}$. The order type of $L$ is the class of all linear orders isomorphic to $L$. We denote by $\mathbf{0 , 1}, \omega$ and $\zeta$ the order type of the order with 0 and 1 element, $(\mathbb{N},<)$ and $(\mathbb{Z},<)$ respectively.

A colored linear order is a mapping from a linear order to a finite set of symbols called colors.

A well-order is a linear order for which every non-empty subordering has a smallest element. The order type of a well-order is called an ordinal. We note 
$\varepsilon_{0}$ the smallest ordinal such that $\varepsilon_{0}=\omega^{\varepsilon_{0}}$. For all $n \geq 0$, we define $\omega \uparrow \uparrow n$ by taking $\omega \uparrow \uparrow 0=1$ and $\omega \uparrow \uparrow(n+1)=\omega^{(\omega \uparrow \uparrow n)}$ for $n \geq 0$. In particular $\varepsilon_{0}=\sup \{\omega \uparrow \uparrow n \mid n \geq 0\}$. An $\omega$-word is a colored linear order of order type $\omega$.

Let $(\Sigma,<)$ be a finite ordered alphabet. We write $\Sigma^{*}$ the set of words over $\Sigma$. We write $u \sqsubseteq v$ if $u$ is a prefix of $v$ and $u \perp v$ if $u \nsubseteq v$ and $v \nsubseteq u$. We denote by $u \wedge v$ the greatest common prefix of $u$ and $v$. The lexicographic order on $\Sigma^{*}$ is defined by: $u<_{\operatorname{lex}} v$ iff $u \sqsubseteq v$ or $u=w a w^{\prime}$ and $v=w b w^{\prime \prime}$ with $a<b \in \Sigma$.

A deterministic tree $t$ over an ordered alphabet $\Sigma$ is a prefix-closed subset of $\Sigma^{*}$. If $u \sqsubseteq v$, we say that $u$ is an ancestor of $v$ or equivalently that $v$ is a descendant of $u$. Elements of $t$ are called nodes and nodes without proper descendant are called leaves. A colored deterministic tree $t$ is a mapping from a deterministic tree $t$ to a finite set of symbols called colors.

A deterministic tree is pruned if it is binary (i.e. $\Sigma=\{0,1\}$ with the usual order), full (i.e. every node is either a leaf or has two sons) and below every node there is at least one leaf.

The frontier of a deterministic tree $t$, denoted $\mathbf{F r}(t)$, is the linear order obtained by considering the leaves of $t$ with the lexicographic order. The colored frontier of a deterministic tree $t$ colored by $\Gamma$ is the mapping from $\operatorname{Fr}(t)$ to $\Gamma$ associating to each leaf of $t$ its color in $t$. In the following, whenever we talk about a deterministic tree we always assume that the alphabet is ordered.

\subsection{Graphs and Monadic Second-Order Logic}

Let $\Sigma$ and $\Gamma$ be two finite sets of arc and vertex labels respectively. Vertex labels are also called colors. A (labeled) graph $G$ is a subset of $V \times \Sigma \times V \cup \Gamma \times V$ where $V$ is a finite or countable arbitrary set. An element $(s, a, t)$ of $V \times \Sigma \times V$ is an arc of source $s$, target $t$ and label $a$, and is written $s \stackrel{a}{\rightarrow} t$ if $G$ is understood. An element $(c, s) \in \Gamma \times V$ intuitively means that $s$ is colored by $c$.

The set of all vertices appearing in $G$ is its support $V_{G}$. A sequence of arcs $s_{1} \stackrel{a_{1}}{\longrightarrow} t_{1}, \ldots, s_{k} \stackrel{a_{k}}{\longrightarrow} t_{k}$ with $\forall i \in[2, k], s_{i}=t_{i-1}$ is a path starting from $s_{1}$. We write $s_{1} \stackrel{u}{\rightarrow} t_{k}$ where $u=a_{1} \ldots a_{k}$.

A graph is deterministic if there are no arcs with the same label that share the same source but not the same target (i.e., for all $a \in \Sigma$, if $s \underset{G}{\stackrel{a}{\longrightarrow}} t$ and $s \underset{G}{\stackrel{a}{\longrightarrow}} t^{\prime}$ then $t=t^{\prime}$ ). A graph $G$ is a tree if there exists a vertex $r$ called the root such that for any vertex in the graph there exists a unique path from the root $r$ to this vertex.

Linear orders and deterministic trees and their respective colored versions can be represented by graphs in a natural way. A linear order $(L,<)$ is represented by the graph $\{(u,<, v) \mid u, v \in L$ and $u<v\}$. A deterministic tree $t$ over $\Sigma$ is represented by the graph $\{(u, a, u a) \mid u, u a \in t$ and $a \in \Sigma\}$. In the following, we will not distinguish between these objects and their graph representations.

We consider monadic second-order (MSO) logic over graphs with the standard syntax and semantics (see e.g. EF95, for a detailed presentation). We write $\varphi\left(X_{1}, \ldots, X_{n}, y_{1}, \ldots, y_{m}\right)$ to denote that the free variables of the formula 
$\varphi$ are among $X_{1}, \ldots, X_{n}$ (monadic second-order) and $y_{1}, \ldots, y_{m}$ (first-order). A formula without free variables is called a sentence.

For a graph $G$ and a sentence $\varphi$, we write $G \models \varphi$ if $\mathcal{G}$ satisfies the formula $\varphi$. The MSO-theory of $G$ is the set of sentences satisfied by $G$. For all formula $\varphi\left(X_{1}, \ldots, X_{n}, y_{1}, \ldots, y_{m}\right)$, all sets $U_{1}, \ldots, U_{n}$ of nodes of $G$ and all nodes $v_{1}, \ldots, v_{m}$ of $G$, we write $G \models \varphi\left[U_{1}, \ldots, U_{n}, v_{1}, \ldots, v_{m}\right]$ to express that $\varphi$ holds in $G$ when $X_{i}$ is interpreted as $U_{i}$ for all $i \in[1, n]$ and $y_{j}$ is interpreted as $v_{j}$ for all $j \in[1, m]$.

\subsection{Graph Transformations}

The unfolding $\operatorname{Unf}(G, r)$ of a graph $G$ from a vertex $r \in V_{G}$ is the tree $T$ s.t. for all $a \in \Sigma, \pi \stackrel{a}{\rightarrow} \pi^{\prime} \in T$ if and only if $\pi$ and $\pi^{\prime}$ are two paths in $G$ starting from $r$ and $\pi^{\prime}=\pi \cdot(s \stackrel{a}{\rightarrow} t)$. Moreover for any color $c \in \Gamma,(c, \pi) \in T$ if and only if $\pi$ is a path in $G$ starting with $r$ and ending in $t$ with $(c, t) \in G$.

An MSO-interpretation is given by a family $\mathcal{I}=\left(\varphi_{a}(x, y)\right)_{a \in \Sigma} \cup\left(\varphi_{c}(x)\right)_{c \in \Gamma}$ of MSO-formulas. Applying such an MSO-interpretation to a graph $G$ we obtain the graph $\mathcal{I}(G)$ labeled by $\Sigma$ and colored by $\Gamma$ and s.t. for all $a \in \Sigma,(u, a, v) \in \mathcal{I}(G)$ iff $G \models \varphi_{a}(u, v)$ and for all $c \in \Gamma,(c, u) \in \mathcal{I}(G)$ iff $G \models \varphi_{c}(u)$.

An MSO-coloring is a particular MSO-interpretation that only affects colors and leaves the arcs unchanged (i.e., for all $a \in \Sigma, \varphi_{a}(x, y)=x \stackrel{a}{\rightarrow} y$ ).

Interpretations cannot increase the size of a structure. To overcome this weakness the notion of a transduction was introduced Cou94. Let $K=\left\{k_{1}, \ldots, k_{m}\right\}$ be a finite set disjoint from $\Sigma$. A $K$-copying operation applied to $G$ adds, to every vertex of $G, m$ out-going arcs labeled resp. by $k_{1}, \ldots, k_{n}$ all going to fresh vertices. An $M S O$-transduction $\mathcal{T}$ is a $K$-copying operation followed by an MSO-interpretation $\mathcal{I}$.

\subsection{MSO on Deterministic Trees}

A non-deterministic tree-walking automaton (TWA) working on deterministic trees over $\Sigma$ colored by $\Gamma$ is a tuple $W=\left(Q, q_{0}, F, \Delta\right)$ where $Q$ is the finite set of states, $q_{0} \in Q$ is the initial state, $F$ is the set of final states and $\Delta$ is the set of transitions. A transition is a tuple $(p, c, q, a)$ with $p \in Q$ and $c \in \Gamma$ - corresponding respectively to the current state and the color of the current node $-q \in Q$ and $a \in(\{\uparrow, \varepsilon\} \cup \Sigma)-q$ being the new state and $a$ the action to perform. Intuitively $\varepsilon$ corresponds to "stay in the current node", $\uparrow$ to "go to the parent node" and $d \in \Sigma$ corresponds to "go to the $d$-son". We say that $W$ accepts a pair of nodes $(u, v)$ if it can reach $v$ in a final state starting from $u$ in the initial state.

Proposition 1 ([Car06, Prop. 3.2.1]). For any deterministic tree $t$ and any $M S O$-formula $\varphi(x, y)$, there exists an MSO-coloring $\mathcal{M}$ and a TWA $\mathcal{A}$ such that $t=\varphi[u, v]$ iff $\mathcal{A}$ accepts $(u, v)$ on $\mathcal{M}(t)$. 


\subsection{The Pushdown Hierarchy}

Following [Cau02, we define the pushdown hierarchy by iterating MSO-interpretations and unfoldings starting from finite graphs.

Tree $_{0}=$ the class of finite trees

$\mathrm{Graph}_{n}=$ the class of graphs MSO-interpretable in a tree of Tree

Tree $_{n+1}=$ the class of the unfoldings of graphs in Graph ${ }_{n}$.

All the graphs in $\mathrm{Graph}_{n}$ have a decidable MSO-theory. Furthermore the hierarchy is unchanged if we require that any graph in $\mathrm{Graph}_{n}$ is MSO-interpreted in a deterministic tree in Tree $_{n}$ [W03. We only recall the two properties that will be used in this article. For a more detailed presentation, we refer the reader to Tho03.

Proposition 2 ([CW03]). For all n, the deterministic trees of Tree $_{n}$ are closed under MSO-coloring and $\mathrm{Graph}_{n}$ is closed under MSO-transduction.

Proposition 3 ([Fra05, Car06]). Take $t$ a deterministic tree in Tree $_{n}, \varphi(X)$ a MSO-formula and $\$$ a fresh color symbol. If $t=\exists X \varphi(X)$ then there exists $U \subseteq t$ s.t. $t \models \varphi[U]$ and $t \cup\{(\$, u) \mid u \in U\}$ also belongs to Tree $_{n}$.

\section{Frontiers of Trees in the Pushdown Hierarchy}

In this section, we show that every (colored) linear order in $\mathrm{Graph}_{n}$ is the (colored) frontier of a pruned tree in Tree ${ }_{n}$. Remark that as the lexicographic order on a deterministic tree is definable in MSO logic, the frontiers of the deterministic trees in Tree ${ }_{n}$ belongs to $\mathrm{Graph}_{n}$. The following theorem establishes the converse inclusion.

Theorem 1. Each colored linear order in $\mathrm{Graph}_{n}$ is the frontier of a colored pruned tree in Tree $_{n}$.

Proof (Sketch). To simplify the presentation, we focus on the uncolored case. Let $L=\left(D,<_{L}\right)$ be a linear order in $\mathrm{Graph}_{n}$ for some $n \geq 0$.

Using the definition of $\mathrm{Graph}_{n}$ and Prop.11and 2, we have that there exists a colored deterministic tree $t \in \operatorname{Tree}_{n}$ and a TWA $\mathcal{A}$ such that $D$ is a set of nodes of $t$ and for all $u, v \in D, u<_{L} v$ iff $\mathcal{A}$ accepts $(u, v)$. We can w.l.o.g. assume that $D$ is the set of leaves of $t$. For instance, it is enough to add a new leaf below every node of $D$ and to modify $\mathcal{A}$ accordingly.

The following construction rearranges the leaves of $t$ into a new deterministic tree $s(t) \in \operatorname{Tree}_{n}$ so that lexicographic order on the leaves of $s(t)$ matches $<_{L}$ on the leaves of $t$. It is easy to adapt the definition of $s(t)$ to obtain a pruned tree (while remaining in Tree $_{n}$ ).

For all leaf $u \in D$ and for all $v \sqsubset u \in D$, we define $s(u, v)$ as the maximal length of a decreasing sequence $u_{0}>_{L} u_{1}>_{L} \ldots>_{L} u_{k}$ of leaves starting with $u_{0}=u$ and such that for $i \in[0, k-1], u_{i} \wedge u_{i+1}=v$. Using a pumping argument on $\mathcal{A}$, we can show that there exists $n_{0} \geq 1$ such that for every leaf $u \in D$ and all nodes $v \sqsubset u, s(u, v) \leq n_{0}$. 
To every leaf $u=u_{1} \cdots u_{n}$, we associate the finite sequence $\boldsymbol{s}(u)$ in $\left[1, n_{0}\right]^{n}$ defined by $s(u)=\left(s(u, \varepsilon), s\left(u, u_{1}\right), \ldots, s\left(u, u_{1} \cdots u_{n-1}\right)\right)$. The key property of this sequence is that comparing two leaves $u$ and $v$ with $<_{L}$ is equivalent to comparing $\boldsymbol{s}(u)$ and $\boldsymbol{s}(v)$ using the lexicographic order, i.e. $u<_{L} v$ iff $\boldsymbol{s}(u)<_{\operatorname{lex}} \boldsymbol{s}(v)$.

Consider the tree $s(t)$ over the finite alphabet $\left[1, n_{0}\right]$ obtained by taking the prefix-closure of $\{\boldsymbol{s}(u) \mid u$ leaf of $t)\}$. The frontier of $s(t)$ is isomorphic to $\left(D,<_{L}\right)$. These definitions are illustrated on the finite example below; $t$ is on the left and $s(t)$ is on the right; for each leaf $x$, we give its order for $<_{L}$ and the sequence $s(x)$.
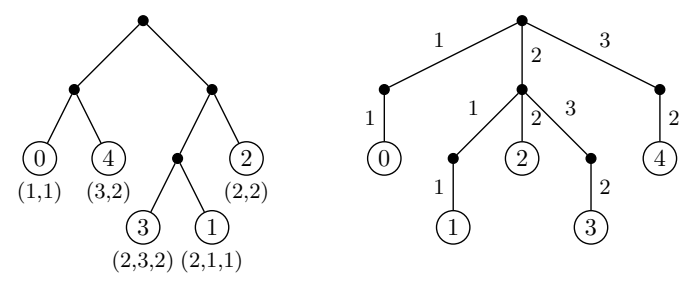

The last step of the proof is to show that $s(t)$ also belongs to Tree ${ }_{n}$.

We know from Cau02 that the infinite terms in $\mathrm{Tree}_{n}$ are the terms solutions of safe recursive schemes of order $(n-1)$ (we refer the reader to KNU02 for a formal definition of safe recursion schemes). Hence the previous theorem can be restated as follows.

Corollary 1. A linear order colored by $\Gamma$ is in $\mathrm{Graph}_{n}$ if and only if it is the colored frontier of some tree solution of a safe recursion scheme of order $(n-1)$ with one terminal $f$ of arity 2 and terminal of arity 0 for each $c \in \Gamma$.

As a first application of this result, we show that $\omega$-words in $\mathrm{Graph}_{2}$ are precisely the morphic words. A morphic word over a finite alphabet $\Gamma$ is given by a letter $\Delta \in \Gamma$ and two morphisms $\tau$ and $\sigma: \Gamma \mapsto \Gamma^{*}$ such that $\tau(\Delta)=\Delta . u$ with $u \in \Gamma^{*}$. The associated $\omega$-word is $\sigma\left(\tau^{\omega}(\Delta)\right)$. For instance, the morphic word abaab $\ldots a^{2^{i}} b \ldots$ is given by the morphisms $\tau$ and $\sigma$ defined by: $\tau(\Delta)=\Delta b a a$, $\tau(a)=a a, \tau(b)=b$ and $\sigma(\Delta)=a, \sigma(a)=a, \sigma(b)=b$.

A direct consequence of [Cau02, Prop. 3.2] is that the morphic words belong to $\mathrm{Graph}_{2}$. For the other direction, by Corollary 1 we only need to consider the frontier of trees solutions of order- 1 schemes. As their frontier are of order type $\omega$, these trees have at most one infinite branch which is also their right-most branch. This constraint leads to a strong normal form for the associated schemes which easily allows to show that their colored frontiers are morphic words.

Theorem 2. The $\omega$-words of $\mathrm{Graph}_{2}$ are the morphic words.

\section{Ordinals}

In this section, we characterize the ordinals in the pushdown hierarchy. In Bra09, ordinals below $\omega \uparrow \uparrow(n+1)$ are shown to be in $\operatorname{Graph}_{n}$. We show that they are the only ordinals in $\mathrm{Graph}_{n}$. 
By Theorem 1 we only need to consider frontiers of pruned trees. Pruned trees with a well-ordered frontier are easily characterized.

Definition 1. A pruned tree is well-ordered if it does not have any infinite branch containing infinitely many 0's.

The frontier of a pruned tree $t$ is an ordinal if and only if $t$ is a well-ordered tree.

Theorem 3. For all $n \geq 0$ and ordinal $\alpha, \alpha \in \mathrm{Graph}_{n}$ if and only if $\alpha<\omega \uparrow \uparrow$ $(n+1)$.

Proof (Sketch). As previously mentioned, we only need to show that for all $n \geq 0$, for any well-ordered tree $t \in \operatorname{Tree}_{n}, \operatorname{Fr}(t)<\omega \uparrow \uparrow(n+1)$.

We proceed by induction on the level $n$. The case $n=0$ is obvious. Let $t$ be a well-ordered tree in Tree ${ }_{n+1}$. By definition of Tree ${ }_{n+1}$, there exists a graph $G \in \operatorname{Graph}_{n}$ and a vertex $r \in V_{G}$ such that $t=\operatorname{Unf}(G, r)$. Furthermore, we can assume w.l.o.g. that every vertex of $G$ is reachable from $r$.

We are going to consider a particular spanning tree $T$ of $G$ rooted at $r$ such that $T$ is MSO-interpretable in $G$.

For every node $s \in V_{G}$, let $\ell(s)$ be the minimal $w \in\{0,1\}^{*}$ (for the lexicographic order) such that $r \underset{G}{\rightarrow} s$. The existence of $\ell(s)$ is ensured by the fact that $G$ unfolds from $r$ into a well-ordered tree. The spanning tree $T$ is defined as the set of $\operatorname{arcs}\{s \stackrel{a}{\longrightarrow} t \mid \ell(t)=\ell(s) \cdot a\}$.

For technical reasons, we consider the pruned tree $\bar{T}$ obtained by adding dummy leaves below some nodes of $T$ in such a way that their out-degree in $\bar{T}$ is equal to their out-degree in $G$. As any infinite branch of $\bar{T}$ is also an infinite branch of $t, \bar{T}$ is a well-ordered.

As $\bar{T}$ can be MSO-transducted in $G$, it belongs to $\mathrm{Graph}_{n}$ together with its frontier. Hence by induction hypothesis, $\operatorname{Fr}(\bar{T})<\omega \uparrow \uparrow(n+1)$.

The key property that allows us to conclude is that $\operatorname{Fr}(t) \leq \omega \operatorname{Fr}(\bar{T})$. To establish this inequality, recall that $\omega^{\operatorname{Fr}(\bar{T})}$ is isomorphic to the set of (nonstrictly) decreasing sequences of ordinals below $\operatorname{Fr}(\bar{T})$ with the lexicographic order. Hence it is enough to show that there exists an injective mapping $\Phi$ from the leaves of $t$ to the finite decreasing sequences of leaves of $\bar{T}$ s.t. $\Phi$ preserves the lexicographic order. Recall that a leaf $u$ of $t$ corresponds to a path $\pi_{u}$ in $G$ starting from $r$. Intuitively $\Phi$ associates to a leaf $u$ of $t$ the sequence of leaves of $\bar{T}$ corresponding to the sequence of $\operatorname{arcs}$ in $G \backslash T$ along the path $\pi_{u}$.
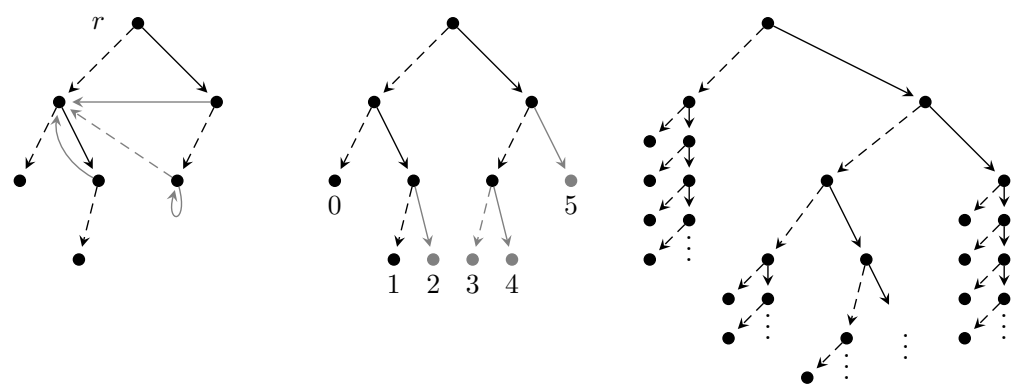
The above example shows from left to right $G$ where dark arcs belong to $T$, then $\bar{T}$ and finally $\operatorname{Unf}(G, r)$. Here, $\operatorname{Fr}(\operatorname{Unf}(G, r))=\omega^{2}+\omega$.

This result gives an alternative proof of the strictness of the pushdown hierarchy and shows that $\varepsilon_{0}$ does not belong to this hierarchy.

\section{$5 \quad$ Scattered Linear Orders}

In this section, we consider the scattered linear orders in the pushdown hierarchy. A linear order is scattered if it does not contain any dense subordering. Ordinals are a particular case of scattered linear orders. However, scattered orders are not necessarily well-orderings; consider for instance $\zeta$ or $\omega+\omega^{*}$. For a detailed presentation, we refer the reader to Ros 82 .

For countable scattered orders, a more constructive characterization is provided by Hausdorff Theorem which also gives a measure of the complexity of such orders. From now on, we only consider countable scattered orders.

Theorem 4 (Hausdorff [Hau08]). A countable linear order is scattered iff it belongs to $\mathcal{S}=\bigcup_{\alpha} V_{\alpha}$ where $V_{0}=\{\mathbf{0}, \mathbf{1}\}$ and $V_{\beta}=\left\{\sum_{i \in \zeta} L_{i} \mid \forall i, L_{i} \in \bigcup_{\alpha<\beta} V_{\alpha}\right\}$.

The Hausdorff rank1 of a scattered order $L$, written $r_{H}(L)$, is the smallest $\alpha$ such that $L$ can be expressed as a finite sum of elements of $V_{\alpha}$. For instance, we have $r_{H}(\zeta)=r_{H}(\omega)=r_{H}\left(\omega+\omega^{*}\right)=1$.

The Hausdorff rank of the ordinal $\omega^{\alpha}$ is equal to $\alpha$. In particular if $\alpha$ is written $\sum_{i=1}^{k} \omega^{\alpha_{i}}$ with $\alpha_{1} \geq \ldots \geq \alpha_{k}$ in Cantor's normal form then $r_{H}(\alpha)=\alpha_{1}$.

\subsection{Trees with Scattered Frontiers}

An alternative characterization of countable scattered orders can be obtained by considering trees having these orders as frontier. The countable scattered orders are those which are frontiers of trees with only countably many infinite branches also called tame trees. The following proposition is part of the folklore.

Proposition 4. Let $t$ be a pruned tree, the following propositions are equivalent:

1. $\operatorname{Fr}(t)$ is a scattered linear order,

2. $t$ has countably many infinite branches,

3. $t$ does not contain any branching subset (i.e. a non-empty subset $U \subseteq t$ such that for all $u \in U, u 0\{0,1\}^{*} \cap U \neq \emptyset$ and $\left.u 1\{0,1\}^{*} \cap U \neq \emptyset\right)$.

The Cantor-Bendixson rank of a tree is an ordinal assessing the branching complexity of a tree. We used a definition taken from KRS05 which is an adaptation of the standard notion Kec94, Exercise 6.172.

\footnotetext{
${ }^{1}$ The standard definition consider the smallest $\alpha$ such that $L$ belongs to $V_{\alpha}$. It is easy to see that this two ranks can only differ by at most one.

${ }^{2}$ See [KRS05, Rem. 7.2] for a comparison of the two notions.
} 
For $X \subseteq t$, we write $d(X)$ for the set of nodes $x \in X$ with at least two infinite branches going through $x$ in $X$. It is easy to see that if $X$ is prefix closed then so is $d(X)$. Hence the operation can be iterated as follows:

$$
d^{0}(X)=X, \quad d^{\alpha+1}(X)=d\left(d^{\alpha}(X)\right), \quad d^{\lambda}(X)=\bigcap_{\alpha<\lambda} d^{\alpha}(X) \text { for limit } \lambda .
$$

The Cantor-Bendixson rank (CB-rank) of $t$, noted $r_{C B}(t)$, is the least ordinal $\alpha$ such that $d^{\alpha}(t)=d^{\alpha+1}(t)$. The tree $t$ is tame if and only if there exists $\alpha$ s.t. $d^{\alpha}=\emptyset$. For tame trees $t$, we adopt a slightly modified version of the CB-rank, denoted $\tilde{r}_{C B}(t)$, which is the smallest ordinal $\alpha$ such that $d^{\alpha}(t)$ is finite. We have $\tilde{r}_{C B}(t) \leq r_{C B}(t) \leq \tilde{r}_{C B}(t)+1$. For pruned tame trees, the CB-rank of the tree and the Hausdorff rank of their frontier are tightly linked.

Proposition 5. For every pruned tame tree $t$,

$$
\begin{cases}\tilde{r}_{C B}(t)=r_{H}(\mathbf{F r}(t)) & \text { if } \tilde{r}_{C B}(t)<\omega \\ \tilde{r}_{C B}(t)=r_{H}(\mathbf{F r}(t))+1 & \text { otherwise. }\end{cases}
$$

As the definition of the CB-rank does not use the relative order between the sons of a node, the CB-rank only depends on the underlying unordered tree. Given two deterministic trees $t$ and $t^{\prime}$, we denote by $t \equiv t^{\prime}$ the fact that $t$ and $t^{\prime}$ are isomorphic when viewed as unordered trees. Formally, $t \equiv t^{\prime}$ if there exists a bijection from $t$ to $t^{\prime}$ preserving the ancestor relation (i.e. for all $u, v \in t, u \sqsubseteq v$ iff $h(u) \sqsubseteq h(v))$.

Proposition 6. For any two pruned tame trees $t$ and $t^{\prime}$, if $t \equiv t^{\prime}$ then $\tilde{r}_{C B}(t)=$ $\tilde{r}_{C B}\left(t^{\prime}\right)$ and $r_{H}(\mathbf{F r}(t))=r_{H}\left(\mathbf{F r}\left(t^{\prime}\right)\right)$.

\subsection{Hausdorff Rank of Scattered Orders in Graph $_{n}$}

From Thm. 3, we know that the pushdown hierarchy is inhabited by scattered orders with a Hausdorff rank $\alpha$ for every $\alpha<\varepsilon_{0}$. This result can be strengthened, by considering the successive powers of $\mathbb{Z}$ defined as follows:

$$
\begin{aligned}
& \mathbb{Z}^{0}=1 \quad \mathbb{Z}^{\beta+1}=\mathbb{Z}^{\beta} \cdot \omega^{*}+\mathbb{Z}^{\beta}+\mathbb{Z}^{\beta} \cdot \omega \\
& \mathbb{Z}^{\lambda}=\left(\sum_{\alpha<\lambda} \mathbb{Z}^{\alpha} \cdot \omega\right)^{*}+1+\sum_{\alpha<\lambda} \mathbb{Z}^{\alpha} \cdot \omega \quad \text { for } \lambda \text { limit. }
\end{aligned}
$$

From [Ros82, Thm. 5.37], the Hausdorff-rank of $\mathbb{Z}^{\alpha}$ is $\alpha$. Furthermore, $\mathbb{Z}^{\alpha}$ is complete among the scattered orders of Hausdorff-rank $\alpha$ in the following sense: a scattered order $L$ has Hausdorff-rank less than $\alpha$ if and only if it is a subordering of $\mathbb{Z}^{\alpha}$.

Proposition 7. For all $n>0$ and any ordinal $\alpha<\omega \uparrow \uparrow n, \mathbb{Z}^{\alpha}$ is in $\operatorname{Graph}_{n}$.

For instance, the following deterministic graph is in $\mathrm{Graph}_{1}$. Its unfolding by the leftmost vertex is in Tree 2 and its frontier is $\mathbb{Z}^{\omega}$. Dashed arcs stand for arcs labeled by 0 , plain arcs stand for for arcs labeled by 1 . 


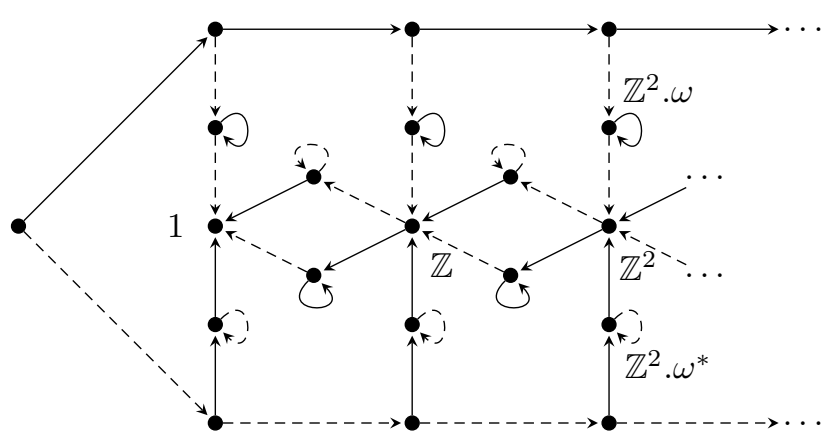

As hinted by the previous proposition, we can show that the Hausdorff rank of any scattered order in $\mathrm{Graph}_{n}$ is strictly less than $\omega \uparrow \uparrow n$. This bound is obtained by reduction to the ordinal case using the following key proposition.

Proposition 8. For any pruned tame treet, there exists a well-ordered tree $t^{\prime}$ such that $t \equiv t^{\prime}$. Furthermore, if $t$ belongs to Tree ${ }_{n}$ then $t^{\prime}$ can also be chosen in Tree $_{n}$.

Proof (Sketch). Consider the following game played by two players Cantor and Hausdorff by moving a token on $t$. The two players play in turn starting with Cantor. Cantor moves the token to a node anywhere below the current position. Hausdorff can only move the token to a son of the current position. Cantor loses the game if the token reaches a leaf.

It is clear that Cantor wins the game if and only if $t$ contains a branching subset. As $t$ is tame (and hence does not contain any branching subset), Cantor loses the game.

From the point of view of Hausdorff this game is a reachability game. Hence Hausdorff has a positional winning strategy: there exists a mapping $\varphi: t \mapsto$ $\{0,1\}$ such that in any play where Hausdorff chooses his moves according to $\varphi$ (i.e. at node $u$, Hausdorff picks the $\varphi(u)$-son) is won by him.

Consider the tree $t_{\varphi}$ obtained from $t$ by swapping the two sons of any node $u$ such that $\varphi(u)=1$. It can be shown that $t_{\varphi}$ is a well-ordered tree : otherwise, from an infinite branch in $t_{\varphi}$ containing infinitely many 0's, we could construct an infinite play in $t$ (i.e. won by Cantor) following $\varphi$.

It remains to prove that $t_{\varphi}$ can be chosen in $\operatorname{Tree}_{n}$ if $t$ is in Tree ${ }_{n}$. A positional winning strategy $\varphi$ can be coded by two sets of vertices $U_{0}, U_{1}$ respectively corresponding to set of nodes $u$ s.t. $\varphi(u)=0$ and $\varphi(u)=1$. Consider an MSOformula $\psi\left(X_{0}, X_{1}\right)$ such that $t \models \psi\left[U_{0}, U_{1}\right]$ if and only if $U_{0}$ and $U_{1}$ encode a positional winning strategy for Hausdorff . Let $c_{0}$ and $c_{1}$ be two color symbols that do not appear in $t$. By Prop. 3, there exist $V_{0}$ and $V_{1}$ such that $t \models \psi\left[V_{0}, V_{1}\right]$ and such that $\bar{t}=t \cup\left\{\left(c_{0}, v\right) \mid v \in V_{0}\right\} \cup\left\{\left(c_{1}, v\right) \mid v \in V_{1}\right\}$ belongs to Tree . $_{\text {. }}$.

The well-ordered tree $t_{\varphi_{0}}$ corresponding to the strategy $\varphi_{0}$ encoded by $V_{0}$ and $V_{1}$ belongs to Tree ${ }_{n}$. As $\bar{t}$ belongs to Tree ${ }_{n}$, there exists a graph $G$ in $\mathrm{Graph}_{n-1}$ and a vertex $r$ of $G$ such that $\bar{t}$ is isomorphic to $\operatorname{Unf}(G, r)$. Consider the MSO-interpretation $\mathcal{I}$ which exchanges the out-going arcs of any vertex colored by $c_{1}$ and erases the colors $c_{0}$ and $c_{1}$. It is easy to check that $\operatorname{Unf}(\mathcal{I}(G), r)$ is isomorphic to $t_{\varphi_{0}}$. 
Theorem 5. For all $n \geq 0$, every scattered linear order in $\mathrm{Graph}_{n}$ has a Hausdorff rank strictly less than $\omega \uparrow \uparrow n$.

Proof (Sketch). Let $L$ be a scattered linear order in $\mathrm{Graph}_{n}$. By Thm. 1 and Prop. 4, there exists a pruned tree $t \in \operatorname{Tree}_{n}$ such that $L \equiv \operatorname{Fr}(t)$. By Prop. 8 there exists a well-ordered tree $t^{\prime} \in$ Tree $_{n}$ such that $t \equiv t^{\prime}$.

By Prop. 6, we have that $r_{H}(\mathbf{F r}(t))=r_{H}\left(\mathbf{F r}\left(t^{\prime}\right)\right)$. As $t^{\prime}$ is a well-ordered tree in $\mathrm{Tree}_{n}$, its frontier is an ordinal in $\mathrm{Graph}_{n}$. Hence by Thm. 3. $\mathbf{F r}\left(t^{\prime}\right)<\omega \uparrow \uparrow n+1$ and hence $r_{H}\left(\mathbf{F r}\left(t^{\prime}\right)\right)<\omega \uparrow \uparrow n$.

Remark 1. Obviously the converse to this theorem is not true; there are uncountably many scattered orders of Hausdorff rank less than $\omega \uparrow \uparrow n$ but there are only countably many linear orderings in $\mathrm{Graph}_{n}$. Consider for instance a non-recursive sequence $\left(a_{i}\right)_{i \in \mathbb{N}}$ in $\{1,2\}^{\omega}$. The scattered order $a_{0}+\zeta+a_{1}+\zeta+a_{2}+\ldots$ has Hausdorff rank 2. But as it has an undecidable MSO-theory, it does not belong to the pushdown hierarchy.

\subsection{Cantor-Bendixson Rank of Deterministic Trees}

By Prop. [5. Thm. 5 can by directly translated on the CB-rank of pruned tame trees in Tree ${ }_{n}$. This leads to the following upper bound for all deterministic trees in Tree $_{n}$.

Theorem 6. For every deterministic tree $t \in \operatorname{Tree}_{n}, r_{C B}(t) \leq \omega \uparrow \uparrow n$.

Proof (Sketch). For every deterministic tree $t \in \mathrm{Tree}_{n}$, there exists a pruned tree $t^{\prime} \in$ Tree $_{n}$ with the same CB-rank. The CB-rank of $t^{\prime}$ is bounded by the supremum of the CB-ranks of the tame subtrees of $t^{\prime}$. As every subtree of $t^{\prime}$ also belongs to $\operatorname{Tree}_{n}, r_{C B}(t)=r_{C B}\left(t^{\prime}\right) \leq \omega \uparrow \uparrow n$.

Acknowledgment. The authors would like to thank the anonymous referees for their helpful comments.

\section{References}

[BÉ07] Bloom, S., Ésik, Z.: Regular and algebraic words and ordinals. In: Mossakowski, T., Montanari, U., Haveraaen, M. (eds.) CALCO 2007. LNCS, vol. 4624, pp. 1-15. Springer, Heidelberg (2007)

[BÉ09] Bloom, S., Ésik, Z.: Scattered algebraic linear orderings. In: Proc. of FICS 2009, pp. 25-30 (2009)

[BÉ10] Bloom, S., Ésik, Z.: Algebraic ordinals. Fundamenta Informaticae (2010) (to appear)

[Blu08] Blumensath, A.: On the structure of graphs in the Caucal hierarchy. TCS 400(1-3), 19-45 (2008)

[Bra09] Braud, L.: Covering of ordinals. In: Proc. FSTTCS 2009, pp. 97-108 (2009)

[Car05] Carayol, A.: Regular sets of higher-order pushdown stacks. In: Jedrzejowicz, J., Szepietowski, A. (eds.) MFCS 2005. LNCS, vol. 3618, pp. 168-179. Springer, Heidelberg (2005) 
[Car06] Carayol, A.: Automates infinis, logiques et langages. PhD thesis, Université de Rennes 1 (2006)

[Cau96] Caucal, D.: On infinite transition graphs having a decidable monadic theory. In: Meyer auf der Heide, F., Monien, B. (eds.) ICALP 1996. LNCS, vol. 1099, pp. 194-205. Springer, Heidelberg (1996)

[Cau02] Caucal, D.: On infinite terms having a decidable monadic theory. In: Diks, K., Rytter, W. (eds.) MFCS 2002. LNCS, vol. 2420, pp. 165-176. Springer, Heidelberg (2002)

[Cou78] Courcelle, B.: Frontiers of infinite trees. RAIRO 12, 319-337 (1978)

[Cou94] Courcelle, B.: Monadic second-order definable graph transductions: A survey. TCS 126, 53-75 (1994)

[CW03] Carayol, A., Wöhrle, S.: The Caucal hierarchy of infinite graphs in terms of logic and higher-order pushdown automata. In: Pandya, P.K., Radhakrishnan, J. (eds.) FSTTCS 2003. LNCS, vol. 2914, pp. 112-123. Springer, Heidelberg (2003)

[EF95] Ebbinghaus, H.D., Flum, J.: Finite Model Theory. Springer, Heidelberg (1995)

[Eng91] Engelfriet, J.: Iterated stack automata and complexity classes. Inf. Comp. 95(1), 21-75 (1991)

[Fra05] Fratani, S.: Automates à piles de piles.. de piles. PhD thesis, Université Bordeaux 1 (2005)

[Hau08] Hausdorff, F.: Grundzüge einer theorie der geordnete mengen. Math. Ann. 65, 435-505 (1908)

[Hei80] Heilbrunner, S.: An algorithm for the solution of fixed-point equations for infinite words. ITA 14(2), 131-141 (1980)

[Kec94] Kechris, A.: Classical Descriptive Set Theory. Springer, Heidelberg (1994)

[KNU02] Knapik, T., Niwinski, D., Urzyczyn, P.: Higher-order pushdown trees are easy. In: Nielsen, M., Engberg, U. (eds.) FOSSACS 2002. LNCS, vol. 2303, pp. 205-222. Springer, Heidelberg (2002)

[KRS05] Khoussainov, B., Rubin, S., Stephan, F.: Automatic linear orders and trees. ACM Trans. Comput. Log. 6(4), 675-700 (2005)

[Mas76] Maslov, A.N.: Multi-level stack automata. Problems Information Transmission 12, 38-43 (1976)

[Ong07] Ong, L.: Hierarchies of infinite structures generated by pushdown automata and recursion schemes. In: Kučera, L., Kučera, A. (eds.) MFCS 2007. LNCS, vol. 4708, pp. 15-21. Springer, Heidelberg (2007)

[Ros82] Rosenstein, J.: Linear orderings. Academic Press Inc., London (1982)

[Tho03] Thomas, W.: Constructing infinite graphs with a decidable MSO-theory. In: Rovan, B., Vojtáš, P. (eds.) MFCS 2003. LNCS, vol. 2747, pp. 113-124. Springer, Heidelberg (2003) 